\title{
Gemcitabine, Oxaliplatin and Paclitaxel (GemPOx) in Patients with Relapsed or Refractory Germ Cell Tumors After High Dose Chemotherapy, A Retrospective Single-Center Experience
}

\author{
Ramazan ACAR ${ }^{1}$, Ismail ERTURK ${ }^{1}$, Halil KIZILOZ ${ }^{2}$, Sezgin OKCELIK ${ }^{2}$, Birol YILDIZ ${ }^{1}$, \\ Musa Baris AYKAN ${ }^{1}$, Gul Sema Yıldıran KESKIN ${ }^{1}$, Galip BUYUKTURAN², \\ Serkan AKIN ${ }^{4}$, Nuri KARADURMUS ${ }^{1}$

\footnotetext{
${ }^{1}$ University of Health Science, Gulhane Faculty of Medicine, Department of Medical Oncology, Ankara

${ }^{2}$ Nevsehir State Hospital, Deparment of Urology, Nevsehir

${ }^{3}$ University of Health Science, Gulhane Faculty of Medicine, Department of Gastroenterology, Ankara

${ }^{4}$ Hacettepe University, Faculty of Medicine, Department of Medical Oncology, Ankara, TURKEY
}

\begin{abstract}
Germ cell tumors are chemosensitive tumors, and patients with metastatic germ cell tumors can be cured even in the metastatic setting. There are limited treatment options for the patients who have refractory or recurrent disease after high dose chemotherapy (HDCT) with autologous stem cell transplantation. We aimed to show real-life data on health outcomes in adult patients with recurrent or refractory germ cell tumor who received the gemcitabine, oxaliplatin, and paclitaxel (GemPOx) combination regimen.We analyzed the data of 33 patients receiving GemPOx regimen after HDCT in our department between January 2016 and January 2020. Primary objectives were overall response rate (ORR), overall survival (OS), and progression-free survival (PFS). The mean age of the study group was $31.5 \pm 8.2$ years. Of the 33 patients, 27 (81.8\%) were men. The median OS and PFS were 16 months (95\% Cl: 7.33-24.7) and 10 months (95\% Cl: 5.1-14.9), respectively. The one-year OS and PFS were 65.7\% and 40\%, respectively. ORR was $42.4 \%$. Toxicity was managable.Our study demonstrates acceptable safety and efficacy of GemPOx regimen for relapsed refractory patients with germ cell tumors. GemPOx remains one of the best determined systemic treatment options for progressive germ cell tumors patients after HDCT.
\end{abstract}

Keywords: Gemcitabine, Oxaliplatin, Paclitaxel, Germ cell tumors, High dose chemotherapy

\section{INTRODUCTION}

Germ cell tumors is the most common cancer in the 15-35 year old men population, although it accounts for only $1 \%$ of all cancers in men. ${ }^{1}$ Germ cell tumors account for $95 \%$ of testicular cancers. Ovarian germ cell tumors are $70 \%$ of ovarian neoplasm in young women between 10-30 years of age. ${ }^{2}$ Germ cell tumors are chemosensitive tumors, and patients with metastatic germ cell tumors can be cured even in the presence of metastatic setting. ${ }^{3}$ The prognosis of germ cell tumors is ultimately de- pendent on the histology, the origin of the tumor, the clinical stage, and the biochemical response following surgery. Overall survival is excellent in the majority of the cases. Metastatic non-seminom germ cell tumors are classified to categories as good, intermediate and poor risk, seminoms are good and intermediate according to International Germ Cell Cancer Collaborative Group (IGCCCG) stratification. ${ }^{3}$ However patients response to treatment in poor risk. Survival outcomes are in-

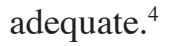


Platinum-based treatments are preferred first in germ cells tumors patients who have advance stage and early stage with risk factors which are vascular or lymphatic invasion, embriyonal carcinoma comprising $>40 \%$ of tumor volume and the presence of yolk sac elements or elevated serum tumors markers prior to orchiectomy or oophorectomy. Although the vast majority of patients can be treated with salvage chemotherapy, the most effective regimen term for these patients is still unclear. ${ }^{5}$ Mostly, high-risk patients have relapsed or refractory disease, and receive additional treatments, including surgery and salvage chemotherapy and even HDCT. ${ }^{6}$ HDCT for consolidation is recommended in cases with relapsed refractory germ cell tumours. ${ }^{7}$

Among the treatment currently available for patients who have relapsed or refractory disease after HDCT are very limited. In these cases, gemcitabine based treatments, such as gemcitabine plus oxaliplatin (GEMOX), gemcitabine, oxaliplatin, and paclitaxel (GemPOx) can be used. ${ }^{8}$ Surgery and second autologous transplantation can also be recommended if the patient is suitable for these treatments. ${ }^{9}$ GemPOx treatment was found very satisfactory in testicular cancer patients who have relapsed or refractory after cisplatin-based chemotherapy. ${ }^{8}$ Besides, Bokemeyer et al. combination chemotherapy with GemPOx has shown to be a good treatment regimen with acceptable toxicity in patients with relapsed or refractory germ cell tumors. ${ }^{10}$

According to the literature most studies in the field of relapsed or refractory germ cell cancer after HDCT are consisted of small case series. ${ }^{9}$ It is still not known about the optimal treatment of these patients .

In this study, we aimed to show real- life data on health outcomes in relapsed or refractory germ cell cancer patients who received the GemPOx combination regimen and to show the ORR, OS and PFS.

\section{PATIENTS AND METHODS}

\section{Patients}

We analyzed the data of 33 patients who underwent HDCT in our department, on account of refractory or relapsed germ cell tumors between 2016 and 2020. All patients were relapsed and underwent various cycles of cisplatin-based and etoposidebased chemotherapy before HDCT. Biochemical markers which are reliable indicators of relapsing disease in germ cell tumors including beta human chorionic gonadotropin ( $\beta \mathrm{HCG})$, lactate dehydrogenase (LDH), and alpha fetoprotein (AFP) were recorded. Demographic and therapeutic details including date of diagnosis, date of recurrence and initial treatments, cycles of chemotherapies, and histologic features of the tumor, toxicity profiles of GemPOx treatment regimen were recorded.

All patients received a standard initial chemotherapy of BEP (comprising bleomycin, etoposide and cisplatin $)^{11}$ and TIP (comprising paclitaksel, ifosfamide and cisplatin) as salvage chemotherapy. ${ }^{5}$ After the salvage chemotherapy, all patients received HDCT.

In this study we chose the patients who had relapsed or refractory disease after HDCT. And all patients recieved GemPOx regimen. Gemcitabine at a dose of $800 \mathrm{mg} / \mathrm{m}^{2}$ and paclitaxel at a dose of $80 \mathrm{mg} / \mathrm{m}^{2}$ those of which at days 1 and 8 and oxaliplatin $130 \mathrm{mg} / \mathrm{m}^{2}$ at day 1 were administered on the basis of a 3-week cycle as GemPOx. ${ }^{12}$

We seek to demonstrate the overall and progression-free survival rate with post-chemotherapy clinical side effects and tolerance of patients receiving GemPOx regimen retrospectively.

The Ethics Committee of University of Health Science, Gulhane Researh and Training Hospital approved the study with 2020-111 ethical committee number at 10 March, 2020. All procedures were according to the Helsinki Declaration of 1975, which revised in 2008, and all procedures were convenient to the ethical standards of the responsible committee on human experimentation (institutional and national).

\section{Statistical Analysis}

We used SPSS v17 (SPSS Inc., Chicago, IL USA) data sheet considering the patients confidentiality. Data was accessible only by the authorized institutional staff and caregivers. Students' t-test was utilized for the test of normal distribution. Demo- 
graphic indices provided in mean values with the standard deviation or median values with $50 \%$ percentile (25\% and $75 \%$ quartile range) as appropriate. Frequencies noted in numbers with percentiles. Survival analysis was conducted utilizing Kaplan-Meier tables and survival plots provided.

\section{RESULTS}

We determined a total of 120 patients with germ cell tumor who underwent autologous stem cell transplantation with HDCT between September 2016 and March 2020. Thirty-three (27.5\%) of the patients had relapsed or refractory disease after HDCT. All of them had received GemPOx treatment after HDCT. The mean age was 31.5 \pm 8.2 years. Twenty-seven $(81.8 \%)$ of the patients were men. The majority of the patients were stage $3 \mathrm{~A}$ or more advanced at the time of the diagnosis $(78.8 \%$, $\mathrm{n}=26$ ). Tumor histology revealed non -seminom in $32(97 \%)$. And Twenty-two (66.6\%) of them were showing mixt germ cell tumor at diagnosis. The majority of the patients were poor risk according to IGCCCG risk stratification (78.7\%).

All cases underwent HDCT following various degrees of salvage chemotherapy both before and after surgery owing to persistent or recurrent disease. Number of lines before GemPOx treatment was more than three lines in all cases (Table 1). The median number of GemPOx treatment cycles was 4 (3-6). Only two patients had a complete response $(6.1 \%), 13$ patients had progression $(39.4 \%)$, overall response rate (ORR) was $42.4 \%$. Seven $(21.2 \%)$ patients had surgery for residual disease. Patients who were underwent surgery also attain complete response. The mean and median follow up time following HDCT, and GemPOx therapy was $10.8 \pm 5.5$ and 10 (3-22) months and 7.3 \pm 4.7 and 6 months, respectively. Twelve patients were died at the follow up period due to the disease progression. None of them were related to the treatment. The median overall survival (OS) and progression free survival (PFS) were after GemPOx treatment; 16 months (95\% CI: 7.33-24.7) and 10 months (95\% CI: 5.1-14.9), respectively (Figure 1 and 2). OS and PFS rates at 12 th month were $65.7 \%$ and $40 \%$, respectively.
Table 1. The demographic and disease-related characteristics of the patients

\begin{tabular}{|c|c|}
\hline Features & n (\%) \\
\hline Mean $\pm S D($ Min-Max) & $31.5 \pm 8.2(18-52)$ \\
\hline Age (years) & $33(100)$ \\
\hline \multicolumn{2}{|l|}{ Gender } \\
\hline Male & 27 (81.8) \\
\hline Female & $6(18.2)$ \\
\hline \multicolumn{2}{|l|}{ Primary origin of tumor } \\
\hline Left testis & $11(33.3)$ \\
\hline Right testis & $16(48.4)$ \\
\hline Ovarian & $6(18.3)$ \\
\hline \multicolumn{2}{|l|}{ Histopathology } \\
\hline Mix germ cell tumor & $22(66.6)$ \\
\hline Yolk sac tumor & $2(6.1)$ \\
\hline Embrional carcinoma & $2(6.1)$ \\
\hline Seminoma & $1(3)$ \\
\hline Choriocarcinoma & $4(12.1)$ \\
\hline Teratoma & $2(6.1)$ \\
\hline \multicolumn{2}{|l|}{ Stage at the Time of Diagnosis } \\
\hline$\geq 3 \mathrm{~A}$ & $26(78.8)$ \\
\hline$\leq 2 \mathrm{C}$ & $7(21.2)$ \\
\hline \multicolumn{2}{|l|}{ Site of metastases } \\
\hline Lung & $9(27.3)$ \\
\hline Lung and Bone & $9(27.3)$ \\
\hline Lung, Liver, Brain and Bone & $8(24.2)$ \\
\hline Only Lymph Node & $7(21.2)$ \\
\hline \multicolumn{2}{|l|}{ IGCCCG Risk Grou } \\
\hline Good Risk & $5(15.2)$ \\
\hline Intermediate Risk & $2(6.1)$ \\
\hline Poor Risk & $26(78.7)$ \\
\hline \multicolumn{2}{|l|}{ Number of lines before GemPOx } \\
\hline$\leq 3$ lines & $15(45.5)$ \\
\hline$>3$ lines & 18 (54.5) \\
\hline \multicolumn{2}{|c|}{ The response of the patients after GemPOx } \\
\hline Stable response & $6(18.2)$ \\
\hline Partial response & $12(36.3)$ \\
\hline Complete response & $2(6.1)$ \\
\hline Progressive disease & $13(39.4)$ \\
\hline
\end{tabular}

Toxicities are listed in Table 2. Leukopenia, anemia, thrombocytopenia, neurotoxicity, febrile neutropenia, diarrhea and vomiting/nausea were observed. One patient had grade 3 febrile neutropenia. No bleeding was observed. Two patients had reversible grade 2 renal toxicity. There was no lifethreatening toxicity. 


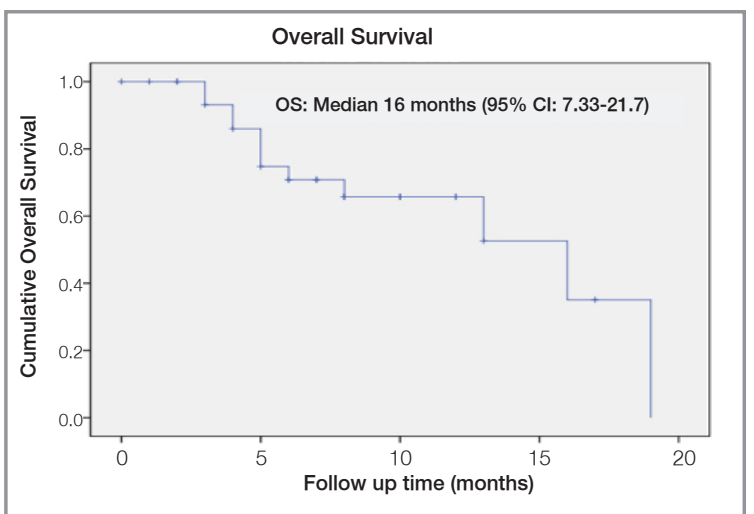

Figure 1. Overall survival of patients recieved GemPOx treatment (Kaplan-Meier Curve)

Biochemical tumor markers before and after GemPOx therapy are shown in Figure 3. Median AlfaFetoprotein (AFP) levels before and after GemPOx were 42.5 (IQR: 8.25-1000) and 12 (IQR: 6.5160), respectively. Median Beta-Human Chorionic Gonadotropin (Beta-HCG) levels before and after GemPOx were 15 (IQR: 0.75-867.5) and 5 (IQR: 0.55-25), respectively. Median Lactate Dehidrogenease (LDH) levels before and after GemPOx were 189 (IQR: 169-620) and 190 (IQR: 150-200), respectively. The decrease in AFP and Beta-HCG levels were as expected. LDH levels were unexpected.

\section{DISCUSSION}

To our knowledge, our study is the first Turkish report of GemPOx regimen after HDCT in patients

\begin{tabular}{|lll|}
\hline \multicolumn{3}{|l|}{ Table 2. Toxicities of GemPOx } \\
\hline & All n (\%) & Grade 3 and 4 n (\%) \\
\hline Leukopenia & $21(63.7 \%)$ & $2(6.1 \%)$ \\
Anemia & $15(45.4 \%)$ & $1(3 \%)$ \\
Thrombocytopenia & $20(60.6 \%)$ & $2(6.1 \%)$ \\
Febril Neutropenia & $7(21.2 \%)$ & $1(3 \%)$ \\
Neurotoxicity & $18(54.5 \%)$ & $4(12.1 \%)$ \\
Vomiting/Nausea & $22(69.7 \%)$ & $3(9.1 \%)$ \\
Diarrhea & $10(30.3 \%)$ & 0 \\
\hline
\end{tabular}
ceptable ORR, OS and PFS. And we showed the efficacy and safety of GemPOx. Most of our patients received HDCT as third-line therapy. In the literature there are serious deficiences about the treatment methods with this patients. After progression, single-agent therapy like oral etoposide, paclitaxel or gemcitabine can be arranged,or patients can be included palliative care according to their situations. ${ }^{13}$ Also, some studies investigated different therapy combinations. ${ }^{12,14}$ We especially chose the patients who were suitable for the combination therapy according to their performance status. We did not include other patients who received singleagent therapy. The GemPOx treatment could not be tolerable for them.

Some studies showed ORRs in the range of 20$44 \% .^{4,12,15}$ Currently, there is no standard salvage

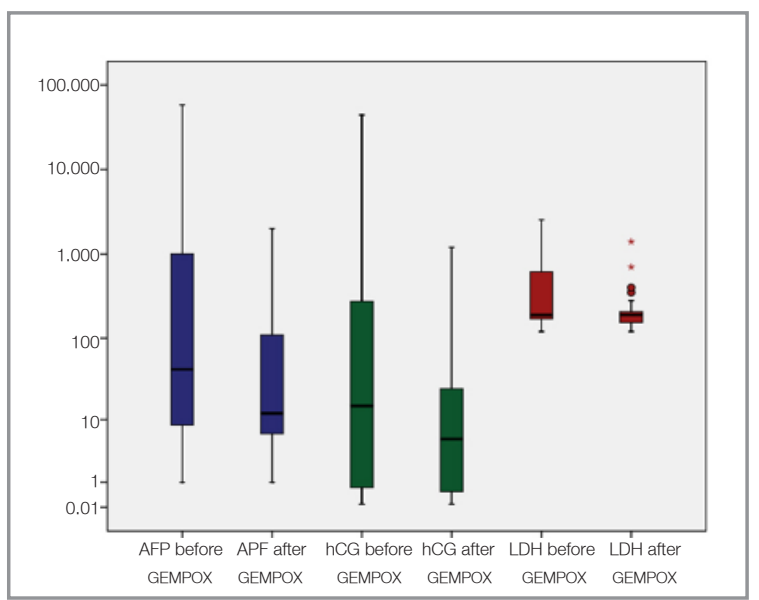

Figure 3. Biochemical serum markers before and after the GemPOx 
chemotherapy for these patients. Seidel et al. observed ORR in $44 \%$ of 63 patients who have received same treatment. ${ }^{12}$ Shiraishi et al. presented a triple-combination regimen using nedaplatin with gemcitabine and paclitaxel. ${ }^{16}$ They observed ORR in $47 \%$ of patients in a small group of patients as 15. We observed ORR in $42.4 \%$ of 33 patients which was similar with literature .

Most of the patients had poor prognosis according to the IGCCCG risk groups. So that the ORR could be acceptable and perfect. Our patients received the GemPOx as a fourth or fifth line therapy after HDCT. This also made the treatment difficult. We trusted the chemosensitivity of germ cell tumors.

Nicolai et al. demonstrated the results of a phase 2 study consists of GemPOx treatment in progressive testicular cancers. They reported 5 year OS $18 \% .{ }^{17}$ Oechsle K. et al. reported that after a median follow-up of 19 months, median OS was eight months for relapsed and refractory germ cell tumor patients. Long term survival was achieved $10-15 \%$ of 76 patients. ${ }^{11}$

Siedal et al. showed the median OS and PFS as 13.3 months and 4 months, respectively. ${ }^{12}$ In our study the median OS and PFS were as 16 months and 10 months, respectively. These results were also perfect and much better than the literature reported before. Thus, GemPOx regimen remains one of the best determined systemic treatment options for progressive germ cell tumors patients even after HDCT.

Safety analysis of this report showed the feasibility of the GemPOx regimen in patients with heavily pretreated germ cell tumors. The most common toxicities were leukopenia, anemia and thrombocytopenia. We did not observe too much grade 3 or grade 4 toxicities. Nicola et al reported that anemia, thrombocytopenia and leukopenia were $41 \%$, $68 \%$ and $68 \%$, respectively. And three patients had grade 3 renal toxicity. ${ }^{17}$ Our results were similar to the literature. ${ }^{17,18}$ None of them was life threatening. In spite of the combination of two potentially neurotoxic drugs, only four patients had grade 3 neurotoxicity. None of the patients died due to treatment toxicities. Additionally, no patients quitted the treatment due to the toxicity. The toxicities were acceptable. Some patients underwent dose reductions and dose delays.
The patients were poor prognosis and all of them underwent HDCT. With this aspect, data demonstrate perfect OS and PFS for further treatment lines. Patients who have received such intensive treatment and relapsed after HDCT are absent in most centers. One of the most important reasons for this is that our center is very experienced in HDCT and there are many cases. It is not easy to find such patients. The only limitation of our study is that it is retrospective. Except for this, very valuable and good results were obtained. We believe that it will be a guide when choosing the treatment methods in this patient group all over the world.

In conclusion, GemPOx treatment remains one of the best determined systemic treatment options for progressive germ cell tumors patients even after HDCT. Also, the GemPOx treatment was associated with good efficacy and tolerability.

\section{REFERENCES}

1. Nigam M, Aschebrook-Kilfoy B, Shikanov S, Eggener S. Increasing incidence of testicular cancer in the United States and Europe between 1992 and 2009. World J Urol 33: 623631, 2015.

2. Zalel Y, Piura B, Elchalal U, et al. Diagnosis and management of malignant germ cell ovarian tumors in young females. Int $\mathrm{J}$ Gynecol Obstet 55: 1-10, 1996.

3. Hanna NH, Einhorn LH. Testicular cancer- discoveries and updates. N Engl J Med 371: 2005-2016, 2014.

4. Einhorn LH, Williams SD, Chamness A, et al. High-dose chemotherapy and stem-cell rescue for metastatic germ-cell tumors. N Engl J Med 357: 340-348, 2007.

5. Kondagunta GV, Bacik J, Donadio A, et al. Combination of paclitaxel, ifosfamide, and cisplatin is an effective second-line therapy for patients with relapsed testicular germ cell tumors. J Clin Oncol 23: 6549-6555, 2005.

6. De Giorgi U, Rosti G, Papiani G, Marangolo M. The status of high-dose chemotherapy with hematopoietic stem cell transplantation in germ cell tumor patients. Haematologica 87: 95104, 2002.

7. Adra N, Abonour R, Althouse SK, et al. High-dose chemotherapy and autologous peripheral-blood stem-cell transplantation for relapsed metastatic germ cell tumors: the Indiana University experience. J Clin Oncol 35: 1096-1102, 2017.

8. Kollmannsberger $\mathrm{C}$, Rick O, Derigs HG, et al. Activity of oxaliplatin in patients with relapsed or cisplatin-refractory germ cell cancer: a study of the German Testicular Cancer Study Group. J Clin Oncol 20: 2031-2037, 2002. 
9. Ertürk I, Yildiz B, Karadurmus N, et al. Retrospective analysis of long-term survival after combination treatment with gemcitabine, oxaliplatin and paclitaxel in patients with refractory or relapsed testicular cancers. Gulhane Med J 60: 145-150, 2018.

10. Bokemeyer C, Oechsle K, Honecker F, et al. Combination chemotherapy with gemcitabine, oxaliplatin, and paclitaxel in patients with cisplatin-refractory or multiply relapsed germcell tumors: a study of the German Testicular Cancer Study Group. Ann Oncol 19: 448-53, 2008.

11. Oechsle K, Kollmannsberger C, Honecker F, et al. Long-term survival after treatment with gemcitabine and oxaliplatin with and without paclitaxel plus secondary surgery in patients with cisplatin-refractory and/or multiply relapsed germ cell tumors. Eur Urol 60: 850-855, 2011.

12. Seidel C, Oechsle K, Lorch A, et al. Efficacy and safety of gemcitabine, oxaliplatin, and paclitaxel in cisplatin-refractory germ cell cancer in routine care- registry data from an outcomes research project of the German Testicular Cancer Study Group. Urol Oncol 34: 168-e21, 2016.

13. Oing C, Seidel C, Bokemeyer C. Therapeutic approaches for refractory germ cell cancer. Expert Rev Anticancer Ther 18: 389-397, 2018.

14. Shamash J, Powles T, Ansell W, et al. GAMEC- a new intensive protocol for untreated poor prognosis and relapsed or refractory germ cell tumours. Br J Cancer 97: 308-314, 2007.

15. Pectasides D, Pectasides M, Farmakis D, et al. Oxaliplatin and irinotecan plus granulocyte-colony stimulating factor as third-line treatment in relapsed or cisplatin-refractory germcell tumor patients: A phase II study. Eur Urol 46: 216-221, 2004.

16. Shiraishi T, Nakamura T, Mikami K, et al. Salvage chemotherapy with paclitaxel and gemcitabine plus nedaplatin (TGN) as part of multidisciplinary therapy in patients with heavily pretreated cisplatin-refractory germ cell tumors. Int J Clin Oncol 14: 436-441, 2009.
17. Nicolai N, Necchi A, Gianni L, et al. Long-term results of a combination of paclitaxel, cisplatin and gemcitabine for salvage therapy in male germ-cell tumours. BJ Int 104: 340-346, 2009.

18. De Giorgi U, Rosti G, Papiani G, et al. Weekly gemcitabine, paclitaxel, oxaliplatin combination chemotherapy in patients with cisplatin-refractory germ cell tumor: preliminary experience. Am J Clin Oncol 27: 457-460, 2004.

\section{Correspondence:}

\section{Dr. Ramazan ACAR}

Saglik Bilimleri Universitesi, Gulhane Tip Fakultesi Tibbi Onkoloji Anabilim Dali Etlik, Kecioren, ANKARA / TURKEY

Tel: (+90-312) 3044157

GSM: (+90-553) 5920541

e-mail: dr_racar@yahoo.com

\section{ORCIDs:}

$\begin{array}{ll}\text { Ramazan Acar } & 0000-0002-3094-899 X \\ \text { Ismail Erturk } & 0000-0001-6835-0988 \\ \text { Halil Kiziloz } & 0000-0002-5523-9209 \\ \text { Sezgin Okcelik } & 0000-0001-6479-9913 \\ \text { Birol Yildiz } & 0000-0001-8920-6467 \\ \text { Musa Baris Aykan } & 0000-0001-7538-9119 \\ \text { Gulsema Yildiran Keskin } & 0000-0001-6858-6974 \\ \text { Galip Buyukturan } & 0000-0003-4781-9737 \\ \text { Serkan Akin } & 0000-0002-7542-9229 \\ \text { Nuri Karadurmus } & 0000-0003-3291-8062\end{array}$

